\title{
Black Holes for Computational Astrophysicists
}

\author{
Douglas Richstone \\ Astronomy Department, University of Michigan, Ann Arbor, MI, 48109, \\ $U S A$
}

\begin{abstract}
.
Supermassive black holes are a ubiquitous feature of galaxy centers. Understanding the problems posed by their formation and co-evolution with their host galaxies is fertile ground for an astrophysicist with a computer.
\end{abstract}

\section{Introduction}

Supermassive black holes (bh) appear to be a standard feature of galaxy centers. Fairly recent evidence shows that their masses can be predicted from the velocity dispersion of the main-body of the bulge of the host galaxy, suggesting either that much of the black hole growth is part of the process of bulge formation or that either the bh or the bulge regulates each others growth.

In this review we focus briefly on the state of the evidence for this correlation, and then survey the proposed mechanisms. Each of these proposals has virtues, but it is, I think, fair to say that even the most promising lack detailed calculations that set them into the context of the popular paradigm for galaxy formation in which baryonic halos condense within dark matter perturbations and merge to form the galaxy bulges we see today. This is a worthy problem for the practitioners of computational astrophysics represented at this meeting.

\section{The State of the Observational Evidence}

We illustrate here the state of the art as reported by Kormendy and Gebhardt (2001). These plots show estimates of central black hole mass from one of three dynamical techniques, versus either the luminosity of the bulge of the host galaxy or the velocity dispersion of the bulge aperture averaged inside $1 R_{e}$ ("effective radius"). These plots reveal a poor correlation between bh mass and host bulge luminosity, and a better one between bh mass and bulge velocity dispersion. The latter relationship has the form

$$
M_{\bullet}=M_{0}\left(\sigma / 200 \mathrm{~km} \mathrm{~s}^{-1}\right)^{\alpha},
$$

with values of $M_{0}=1.2 \times 10^{8} M_{\odot}$ and $\alpha=3.75$ obtained by Gebhardt et al. (2000) and a similar but steeper relationship given by Ferrarese \& Merritt (2000). We can see no evidence in these data sets, either for a systematic error associated with the method used to estimate the bh mass, or for a "second parameter" involving the Hubble type of the host galaxy. 
We have failed to detect a bh in only one well studied galaxy - M33. The upper mass limit obtained by Gebhardt et al. (2001) was $1500 M_{\odot}$ while Merritt, Ferrarese \& Joseph (2001) got a limit of $3000 M_{\odot}$ from the same data. Since M33 is a bulgeless galaxy this result is not surprising.

These detections and the $M_{\bullet}-\sigma$ relationship constitute a de-facto estimate of the local mass density of supermassive $\mathrm{BH}$, based on the assumption that these observed well-studied galaxies are typical of all galaxies. Estimates of this quantity historically (in units of $1 \times 10^{6} M_{\odot} \mathrm{pc}^{-3}$ ) are 1.0 (Kormendy \& Richstone 1995), 3.0 (Magorrian et al. 1998), 0.5 (Merritt \& Ferrarese 2001 ) and 0.6 (Aller \& Richstone 2001).

The first two results above use the $M_{\bullet}-L_{\text {bulge }}$ relationship together with the luminosity density of galaxies to estimate the mass density of bh. The third paper uses the $M_{\bullet}-\sigma$ relationship to calibrate a $M_{\bullet}-L$ relationship for galaxies, and then uses the luminosity density of galaxies to estimate the mass density of bh. The fourth estimate uses published data to estimate a "velocity dispersion function" for galaxies and directly combines that with the observed $M_{\bullet}-\sigma$ relationship to estimate a mass density of quasars.

This number can be compared to the "Soltan Number", the redshift-corrected mass density of the quasar energy. The Chokshi - Turner estimate of this is obtained from the integrated comoving energy density in quasar light,

$$
u=\int_{0}^{\infty} \int_{0}^{\infty} L \Phi(L \mid z) d L \frac{d t}{d z} d z=1.3 \times 10^{-15} \mathrm{erg} \mathrm{cm}^{-3}
$$

where $\Phi$ is the comoving density of quasars of luminosity $L$, and $t$ is cosmic time. The corresponding present-day mass density for a radiative efficiency $\epsilon$ is

$$
\rho_{u}=u /\left[\epsilon c^{2}\right]=2 \times 10^{5}\left(1-.1 \epsilon_{0.1}\right) \epsilon_{0.1}^{-1} M_{\odot} \mathrm{Mpc}^{-3} .
$$

where we have parameterized the radiative efficiency in terms of $\epsilon_{0.1}=\epsilon / 0.1$ because popular geometrically-thin optically-thick accretion disk models rarely exceed efficiencies of $\epsilon_{0.1}=1$. The (often omitted) factor of $1-\epsilon$ reflects the fact that energy emitted from the accretion flow does not find its way into the relic bh. The "Soltan Number" is independent of Hubble constant or of cosmological model.

It is sometimes useful to compare the bh density to the luminous density in galaxies, $j=1.1 \times 10^{8} L_{\odot} \mathrm{Mpc}^{-3}$ (Loveday et al. 1992), to obtain the ratio of the mass in relic $\mathrm{MBHs}$ to the light of galaxies:

$$
\Upsilon_{\bullet}=\frac{\rho_{u}}{j}=1.8 \times 10^{-3}\left(\frac{0.1}{\epsilon}\right)\left(\frac{M_{\odot}}{L_{\odot}}\right) .
$$

This quantity is not independent of the Hubble constant. It is given here for $H=80 \mathrm{~km} \mathrm{~s}^{-1} / \mathrm{Mpc}$.

In retrospect it is clear that the Magorrian estimate is too high as a result of the assumption of two-integral velocity distributions in the analysis of groundbased data of varied quality. Leaving aside this rather high number the others seem fairly consistent with each other, and with the Chokshi-Turner estimate of the "Soltan Number".

There are several important inferences that follow from the above terse summary of observations, together with the noted assumptions. 

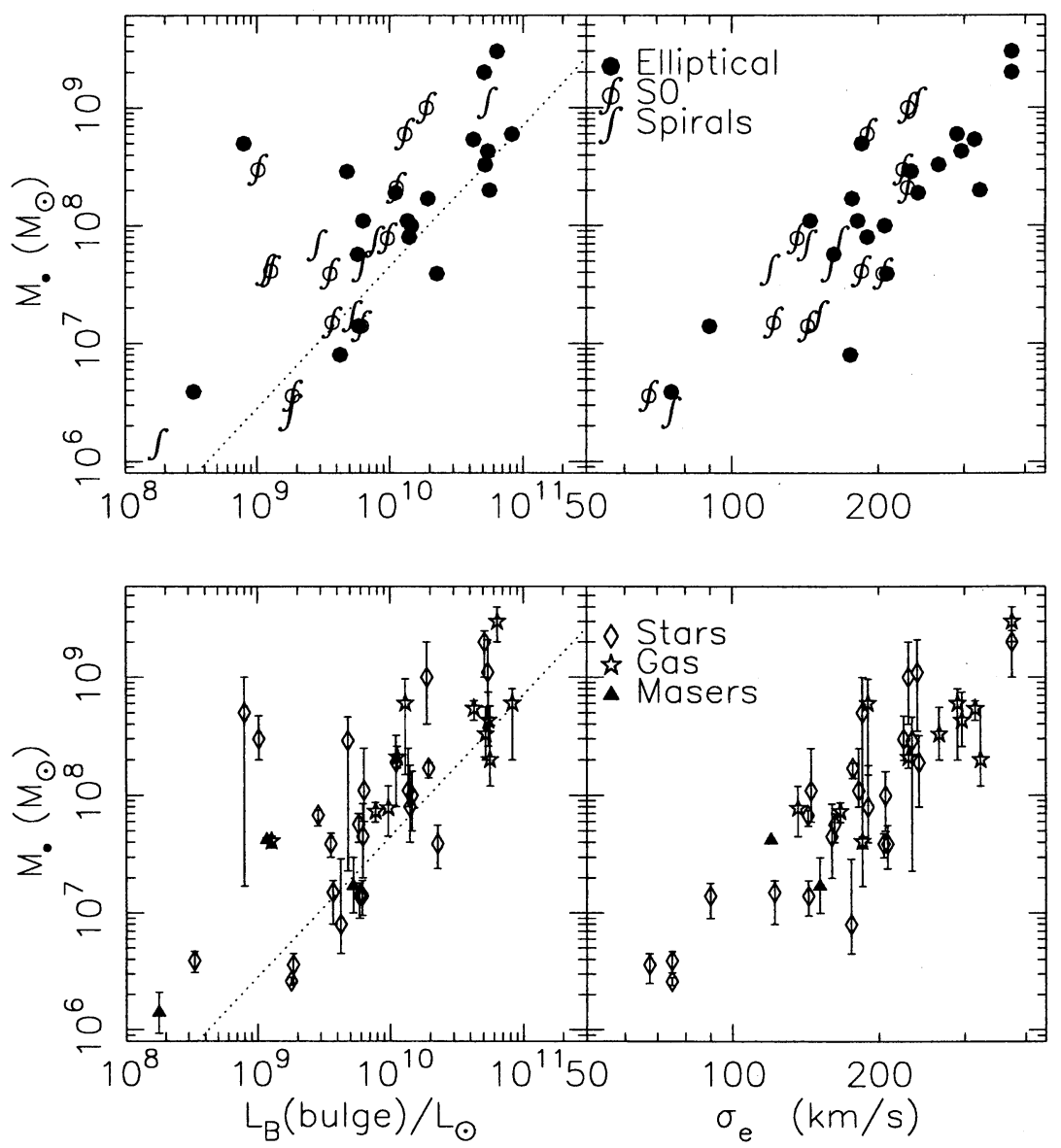

Figure 1. Estimated black hole mass plotted against bulge luminosity (left panels) and bulge root-mean-square velocity (right panel). The upper panels are plotted without estimates in the errors of the bh mass, and with a symbol indicating the Hubble type of the host galaxy. The lower panels are shown with error bars and with a symbol that indicates the method used to compute the bh mass - diamonds for stellar dynamics, stars for gas dynamics and triangles for MASER sources. The dotted line in the left panel is derived from the "Soltan Number" (see text). 
1. Members of the supermassive black hole population are the relics of the quasars (they have the correct masses, density and location).

2. Most of their growth occurred while in the quasar phase and was visible (this doesn't preclude mergers of these objects, which change the numbers and masses but not their cosmic mass density).

3. Even during the quasar epoch the black holes are quite massive because they must exceed their Eddington masses defined by

$$
L_{E}=1.3 \times 10^{46} M_{8} \mathrm{ergs} / \mathrm{sec},
$$

where $L_{E}$ is the Eddington luminosity and $M_{8}$ is the black hole mass in units of $10^{8} M_{\odot}$. Typical bright quasars reach or exceed luminosities of $10^{46} \mathrm{ergs} / \mathrm{sec}$.

4. Reaching this mass is a challenge because because the "Salpeter time" $t_{S}$ (for an Eddington-limited accreting black hole to e-fold in mass) is

$$
t_{S}=M / \dot{M}=4 \times 10^{7} \epsilon_{0.1} \text { yrs. }
$$

5. It is possible that seed black holes (conceivably with a significant fraction of the eventual bh mass) formed much earlier.

6. Becuase the velocity dispersion at $R_{e}$ is closely related to the binding energy per unit mass of the bulge (which is, inside that radius, dominated by visible matter), and is set during bulge formation or subsequent mergers, the growth of the black hole and the formation of its host bulge are physically coupled.

Finally, we reiterate here a minor dilemna first described in Richstone et al. 1998. While the estimate of the density of mass in black holes is consistent with the total energy radiated by the quasars (the integrals over our best guess for the mass function of the former and luminosity function of the latter are roughly equal), there is an excess in the number of bh heavy enough to produce the brightest quasars. At the peak of the quasar epoch there were about $10^{-6} \mathrm{Mpc}^{-3}$ quasars brighter than $6 \times 10^{46} M_{8}$ ergs/sec. It seems reasonable that these brightest quasars are emitting near their Eddington limit, and hence their engines have masses of at least $4 \times 10^{8} M_{\odot}$. Bulges this massive have a local density of $10^{-3} \mathrm{Mpc}^{-3}$. Why are there so few quasars? One can imagine that quasars simply have very short duty cycles, and so the objects are all there but flash only briefly, but this would imply only a small fraction of there mass gain is visible, and that returns us to the integral constraint, which then becomes a coincidence. One possibility suggested is that the bh population has undergone a few mergers since the quasar era. In that case we now observe a mass function, which, while having about the same mass density, has a larger number of rather massive objects than existed at the time of the quasars. 


\section{A Laundry List of Theories for the $M_{\bullet}-\sigma$ Relationship}

The $M_{\bullet}-\sigma$ relationship has stimulated considerable theoretical effort yielding a collection of mechanisms. Many of these can be organized into a crude taxonomy as follows.

\subsection{Models in which $\mathrm{BH}$ growth is limited by a mass budget}

The best example of models that fall into this category is due to Burkert and Silk 2001. They suggest that the mass accreted by the black hole is proportional to the gas mass in the bulge given by

$$
M \propto f_{g} \sigma^{3} \rho^{-1 / 2} \propto f_{g} \sigma^{3}(1+z)^{-3 / 2}
$$

where $f_{g}$ is the mass fraction, $\sigma$ is the velocity dispersion and $z$ is the formation redshift. They suggest that the accretion rate varies as the available gas mass divided by the viscous time, and further argue that the viscous time, the dynamical time and the star formation time are all the same within factors of order unity. In this model the black hole accretes a fixed fraction of all the gas within its cusp radius for one dynamical time (after which the remaining gas will have all formed stars). This model leads to

$$
M_{\bullet} \propto \sigma^{3}
$$

with the scatter coming from variance in the epoch of formation.

This model (and others like it) have two virtues. First, it is unavoidable that star formation in the bulge will compete with the bh for the available gas. Second, it offers a possible physical mechanism that might provide the coupling between $M_{\bullet}$ and $\sigma$ required by the semi-analytic models of Haehnelt \& Kaufman (2000).

\subsection{Models in which $\mathrm{BH}$ growth is limited by a momentum budget}

A straightforward example of this sort of model is due to Silk \& Rees 1998. They argue that some fraction of the luminosity of the quasar exerts a force on the accreting gas, and that this "mechanical luminosity" $f_{w} L$ (a force times distance per crossing time) must be less than the force needed to drive the the gas in the galaxy to escape velocity (of order $\sigma$ ) in a crossing time, so that

$$
f_{w} L \leq f_{g} M_{b u l g e} v^{2}(r / v)^{-1} .
$$

Since $M_{\text {bulge }} \propto \sigma^{2} r$ and L (by assumption) is $L_{E}$ this model predicts

$$
M_{\bullet} \leq\left(\frac{f_{g}}{f_{w}}\right) \sigma^{5} \text {. }
$$

Blandford (1999) has taken this sort of feeback model a step further by suggesting that the energy and momentum radiated by the central black: hole determines the Hubble type of the galaxy. 


\subsection{Models in which BH growth is limited by an energy budget}

In models in which bh formation is a result of core collapse driven by dynamical relaxation and evaporation, conservation of energy is a severe constraint. Because "evaporated" stars carry away approximately zero energy, the total energy of the evolving system doesn't change, it is simply concentrated in a decreasing number of more tightly bound stars. In order for part of the system to collapse relativistically, it's binding energy must approach $M_{\bullet} c^{2}$, while the working reservoir of negative energy is no greater than $M_{t} \sigma^{2}$, where $M_{t}$ is the mass of this reservoir. Since the relaxation time is only short near the center of this mass is surely less than the bulge mass of the galaxy. Equating these energies gives

$$
\frac{M_{\bullet}}{M_{t}} \leq(\sigma / c)^{2} \sim 0.5 \times 10^{-6},
$$

where the numerical estimate is based on taking a velocity dispersion of $200 \mathrm{~km} / \mathrm{s}$ for a typical bulge or elliptical galaxy. Masses of observed black holes are roughly $10^{-3}$ of the bulge mass, so this sort of model clearly needs help. Various participants at the conference suggested that additional energy could be extracted by gravitational radiation. That could certainly work in principle, but even getting to that stage by this method seems challenging (a more optimistic view of a variant of this approach is given in Ebisuzaki et al. 2001).

\subsection{Models which do not fit in this taxonomy}

Two other representative models for bh formation do not fit easily into this taxonomy. Ostriker (2000) has proposed a model in which self-interacting dark matter is accreted by a seed black hole following the Bondi formula, and shows that the model leads to $M \propto \sigma^{4}$ provided the bh is in the center of a spherical singular isothermal mass distribution $\left(\rho \propto r^{-2}\right)$.

A second such model is based on direct infall from a cold mass distribution with $\rho \propto r^{-2}$ and constant angular velocity onto a seed black hole (Adams, Graff, Richstone 2001). In this model the matter can be either non-interacting or baryonic (so long as it is cold). The mass not captured by the bh forms the bulge. If the mean-square angular momentum of the bulge sets the angular velocity, the model "predicts" $M \propto \sigma^{4}$ with the correct zero point. There are two variants of this model, one based on ballistic orbits of dark matter, the other depending on the collapse of a singular isothermal gas sphere. The latter is more promising as noted below.

An important limitation of each model is their possible fragility to changing the assumed initial conditions. This could be further investigated through numerical simulation. A second concern (quite apart from the question of whether the dark matter is self-interacting), is the question of reproducing the "Soltan Number". If a large fraction of bh mass is built up by accretion of dark matter (which produces no luminosity), then we would not expect the observed near equality of the energy density of quasar radiation and the mass density of relics.

\section{The Mutual Evolution of Galaxies and Black Holes}

The quasar epoch peaks at $2 \leq z \leq 3$, a lookback time of $7.2 h^{-1} \mathrm{Gyr}$ to $8 h^{-1} \mathrm{Gyr}$ in a cosmology with $\Omega_{0}=0.3, \lambda=0.7$, so the most conservative approach 
would be to regard supermassive black holes as a key constituent of the galaxy formation and evolution problem since this time. We think it is a good bet that the quasar epoch is also the epoch of major bulge-building (this is not inconsistent with modern views of galaxy formation), and of black hole growth. This view mandates at least 3 kinds of investigations .

\subsection{Galactic and pregalactic mergers}

In a hierarchical theory for the formation of bulges, mergers play a critical role. For much, if not all, of this merger history one or both merger partners is likely to contain a supermassive bh. Most of the famous calculations of mergers do not include a central supermassive bh carrying $0.1 \%$ of the visible mass of the galaxy (indeed, most calculations do not include gas). There are several puzzles that can be addressed by theorists.

1. There is a well known dichotomy between low luminosity ellipticals and bulges and high luminosity ones. Low luminosity galaxies rotate rapidly enough to be flattened by rotation, tend to have disky isophotes and have "cuspy" centers. High luminosity galaxies are flattened by velocity anisotropy, tend to have boxy isophotes and have more nearly analytic cores (there is hot related debate [represented in the papers by Navarro and Weinberg in this volume] about whether CDM predicts galaxy centers that are too cuspy). Faber et al. 1997 have suggested that the softer cores of high luminosity galaxies are scoured out by the decay of the binary black hole during mergers. That model requires different relative histories of star formation and merging for high and low luminosity galaxies (low L galaxies must complete most of their mergers while still gas rich). An important result of simulations of this process by Quinlan and Hernquist (1997) is the prediction of tangentially biased anisotropy of the stellar distribution function near the center of the merger product.

2. The galaxies remain on a fundamental plane. On the plane, small galaxies are much denser than large ones. Lauer et al. (1995) showed that this density contrast exceeds $10^{3}$ over a factor of 100 in galaxy luminosity. The cannibalization of smaller galaxies should therefore result in much higher density cores in the centers of large galaxies, and should destroy the fundamental plane. Weinberg 1997 and Holley-Bockelmann and Richstone (1999, 2000) have investigated this. The results of those studies show that massive bh in the larger galaxies break up the smaller galaxies. Depending on the orbit of the smaller galaxy when it enounters the center of the larger galaxy, it may produce a stellar disk or torus at the center of the larger galaxy.

3. The merger of supermassive black holes is a theoretical challenge. During the merger of two galaxies or protogalaxies, the two galaxies eventually lose their distinct identities. At this point the two black holes sink toward the center of the merger product under the influence of dynamical friction, eventually forming a binary. As this binary hardens its orbital decay slows, until gravitational radiation becomes an important energy loss mechanism. For purely stellar dynamical galaxies and for an interesting range of bh mass, the decay time exceeds a Hubble time. Key features of this problem were laid out in a different context by Hut and Rees (1992) and Ostriker and Xu (1994). Recent calculations are due to $\mathrm{Yu}(2001)$. 


\subsection{The destruction of triaxial structure}

It has long been suggested that the growth of a bh destroys the regularity of phase space in triaxial potentials and thereby renders the box orbits critical for maintenance of the triaxial structure chaotic (Gerhard \& Binney 1985; Hasan \& Norman 1990). Recent papers by Poon \& Merritt (2001) and Holley-Bockelmann et al. (2001) address the question of how far beyond the cusp radius of the bh this effect can symmetrize the structure of the galaxy.

\subsection{The thermal influence of the AGN}

Note that for most of the galaxies plotted in the left panels of figure 1 the ratio of black hole mass to stellar light $\Upsilon$. exceeds $10^{-3}$. At the present epoch, a quiescent bh contributes a negligible fraction of it's host's luminosity. However, when they are on, we can use equation 5 to write their contribution to the galactic energy budget as

$$
\begin{aligned}
\frac{L(b h)}{L(\text { stars })}=3 & \times 10^{4} \Upsilon \bullet\left(\frac{L(b h)}{L_{E}}\right) \\
= & 30\left(\frac{L(b h)}{L_{E}}\right) .
\end{aligned}
$$

We have used the present day value of $\Upsilon_{\bullet}$ in the equation above. It is likely to be smaller at earlier times due to passive evolution of the stellar population. We note, however, that for quasars operating near even $0.03 L_{E}$, the luminous output will rival that from the stars, and the quasars will dominate the X-ray budget in the ISM, especially near the center of the protogalaxy, and will heavily influence the thermal balance of the interstellar gas. Ciotti \& Ostriker (1997 \& 2001) provide one example of the possible consequences of this energy input the heating and disruption of a cooling flow.

Acknowledgments. I'm grateful to my NUKER colleagues for years of illuminating discussions and to Piet Hut and Jun Makino for their patient efforts to get me to write this report.

\section{References}

Adams, F. C., Graff, D. S. \& Richstone, D. O. 2001, ApJ 551, L31.

Aller, M. \& Richstone, D. 2002, in preparation.

Blandford, R. D. 1999, in "Galaxy Dynamics" ed Merritt, Valluri \& Sellwood, also astro-ph 9906025.

Burkert, A. \& Silk, J. 2001, ApJ 554, L151.

Ciotti, L. \& Ostriker, J. P. 1997 ApJ 487, L105.

Ciotti, L. \& Ostriker, J. P. 2001 ApJ 551, 131.

Ebisuzaki, T., Makino, J., Tsuru, T.G., Funato, Y., Portegies Zwart, S.F., Hut, P., McMillan, S.L.W., Matsushita, S., Matsumoto, H., and Kawabe, R., T. 2001 ApJ 562, L19. 
Faber, S. M., Tremaine, S., Ajhar, E, A., Byun, Y.-I., Dressler, A., Gebhardt, K., Grillmair, C., Kormendy, J., Lauer, T. R., and Richstone, D., 1997 AJ 114, 1771.

Ferrarese, L., \& Merritt, D., 2000 ApJ Letters 539, L9.

Gebhardt, K., Bender, R., Bower, G., Dressler, A., Faber, S. M., Filippenko, A. V., Green, R., Grillmair, C., Ho, L. C., Kormendy, J., Lauer, T. R., Magorrian, J., Pinkney, J., Richstone, D., and Tremaine, S., 2000, ApJ Letters 539, L13.

Gebhardt, K., Lauer, T. R., Kormendy, J., Pinkney, J., Bower, G. A., Green, R., Gull, T., Hutchings, J. B., Kaiser, M. E., Nelson, C. H., Richstone, D., and Weistrop, D., 2001, AJ 122, 2469.

Gerhard, O. E. \& Binney, J. 1985 MNRAS 216, 467.

Haehnelt, M. G. \& Kauffmann, G., MNRAS 318, L35.

Holley-Bockelmann, K., Mihos, J. C., Sigurdsson, S. Hernquist, L. and Norman, C. 2001, ApJ in press.

Hasan, H. \& Norman, C. 1990 ApJ 361, 69.

Holley-Bockelmann, K. \& Richstone, D. 1999, ApJ 517, 92.

Holley-Bockelmann, K. \& Richstone, D. 2000, ApJ 531, 232.

Hut, P., Rees. M. 1992, MNRAS 259, 27P.

Kormendy, J., \& Gebhardt, K. in The 20th Texas Symposium on Relativistic Astrophysics, ed. H. Martel \& J.C. Wheeler, in press, and astro-ph/0105230.

Kormendy, J. \& Richstone, D. 1995, Ann Rev Astron and Astroph 33, 581.

Lauer, T. R., Ajhar, E. A., Byun, Y.-I., Dressler, A., Faber, S. M., Grillmair, C., Kormendy, J., Richstone, D., and Tremaine, S., 1995 AJ, 110, 2622.

Loveday, J., Peterson, B. A., Efstathiou, G., and Maddox, S. J., 1992, ApJ 390, 338.

Magorrian, J., Tremaine, S., Richstone, D., Bender, R., Bower, G., Dressler, A., Faber, S. M., Gebhardt, K., G., Grillmair, C., Kormendy, J., and Lauer, T., 1998, AJ 115, 2285.

Merritt, D. Ferrarese, L. 2001, MNRAS 320, L30.

Merritt, D., Ferrarese, L. \& Joseph, C. 2001, Science, 293, 1116.

Ostriker, J. P. 2000, Phys. Rev. Lett, 84, 5258.

Ostriker, J. P. 1994, ApJ, 437, 184.

Poon, M. Y. \& Merritt, D. 2001, ApJ, 549192.

Quinlan, G. \& Hernquist, L. 1997, NewA, 2, 533.

Richstone, D., Ajhar, E. A., Bender, R., Bower, G., Dressler, A., Faber, S. M., Filippenko, A. V., Gebhardt, K., Green, R., Ho, L. C., Kormendy, J., Lauer, T. R., Magorrian, J., and Tremaine, S., 1998, Nature, 395, A14.

Silk, J. \& Rees, M. 1998, A\&A, 331, L1. 
Weinberg, D. 1997, ApJ, 478, 435.

Yu, Q., 2001, MNRAS in press and astro-ph 0109530. 